\title{
Perioperative management of patients with diabetes mellitus: ketone screening
}

\author{
Martin M. Muermann, BSc (Hons) (1) - Petrus J. Retief, MD
}

Received: 10 January 2021/Revised: 25 January 2021 / Accepted: 9 February 2021 / Published online: 23 February 2021

(C) Canadian Anesthesiologists' Society 2021

\section{To the Editor,}

Diabetes mellitus (DM) presents unique challenges to anesthesiologists in perioperative patient management. While guidelines have long relied on serial serum glucose monitoring, updated recommendations for anesthesiologists in pediatric patients with type 1 diabetes ${ }^{1}$ have only recently included ketone screening as part of preoperative patient evaluations. To date, however, this has not been extended to protocols for adults with DM.

Ketone testing has only been mentioned in the literature, if at all, in relation to emergent surgeries or confirming a diagnosis of diabetic ketoacidosis (DKA) in patients who are already symptomatic. Preoperative serum ketone screening for patients with DM may prove useful as a cost-effective form of secondary prevention in diabetic emergencies such as DKA, potentially allowing for earlier detection and intervention. Furthermore, ketone testing is a more accurate marker of intracellular hypoglycemia, as serum glucose levels do not necessarily reflect insulin receptor function, metabolic stress, or ensuing starvation leading to catabolic metabolism.

Flaws in relying primarily on serum glucose testing are evidenced by growing reports of perioperative euglycemic DKA that elude detection by current protocols. ${ }^{2}$ While such cases are mostly associated with sodium-glucose cotransporter-2 inhibitor (SGLT2i) medication use (which has only recently been addressed by the Food and Drug

M. M. Muermann, BSc (Hons) ( $₫)$

School of Medicine, University of British Columbia, Vancouver, BC, Canada

e-mail: martin.muermann@gmail.com

P. J. Retief, MD

Chilliwack General Hospital, Chilliwack, BC, Canada
Administration), ${ }^{3}$ it may take quite some time for established practice standards to catch up. The dramatic increase in popularity of SGLT2i usage and the recent announcement of dapagliflozin use for non-diabetic purposes $^{4}$ indicate that preoperative ketone screening could potentially go a long way towards improving perioperative care.

Without large-scale data, ideally from large, randomized trials of ketone screening, it is not known whether serum ketone screening improves morbidity and/or mortality of patients with type 1 (T1D) or type 2 diabetes (T2D). While screening may logically improve the accuracy of assessing patient insulin demand and reduce physiologic stress in the perioperative period, this may also only lead to an optimization of care rather than a seismic shift forward. Our suspicion is that patients with T2D currently rely on what little endogenous insulin they still produce to withstand the physiologic stress of surgery when receiving less exogenous insulin than required. This might explain why seemingly little attention has been afforded to this subject to date. In comparison, patients with T1D are likely to benefit more strongly from ketone screening as they typically do not possess endogenous stores of insulin and suffer more under comparable stress in the perioperative period.

Diabetic crises such as DKA are a large burden to the healthcare system costing significant resources. Patients with DM who develop such an emergency and who are not detected quickly may require extended hospital stays in intensive care units (ICUs) and may suffer neurologic sequelae or death. Since serum ketone testing is relatively inexpensive, has a high specificity and sensitivity, and point-of-care bedside meters are currently available, ${ }^{5}$ it may improve care while simultaneously reducing burden 
on ICUs currently trying to cope with the ongoing coronavirus disease pandemic.

Additional research into this area is required to determine whether preoperative ketone screening is costeffective and potential improvements to care have a significant effect on morbidity and mortality of adult patients with DM. We encourage debate and deeper investigation into this issue as there are only scarce data presently available.

Disclosures None.

Funding statement None.

Editorial responsibility This submission was handled by Dr. Philip M. Jones, Deputy Editor-in-Chief, Canadian Journal of Anesthesia.

\section{References}

1. Martin LD, Hoagland MA, Rhodes ET, Wolfsdorf JI, Hamrick JL. Perioperative management of pediatric patients with type 1 diabetes mellitus, updated recommendations for anesthesiologists. Anesth Analg 2020; 130: 821-7.

2. Lane S, Paskar D, Hamed S, Goffi A. When guidelines fail: euglycemic diabetic ketoacidosis after bariatric surgery in a patient taking a sodium-glucose cotransporter-2 inhibitor: a case report. A A Pract 2018; 11: 46-8.

3. Franki L. FDA Says Stop SGLT2 Inhibitors for Diabetes Prior to Surgery. Medscape; 2020 Mar 18; Available from URL: https:// www.medscape.com/viewarticle/927047 (accessed January 2021).

4. Melillo G. FDA Approves Dapagliflozin to Treat Heart Failure, Breaking New Ground in SGLT2 Competition. The American Journal of Managed Care; 2020 May 06; Available from URL: https://www.ajmc.com/view/fda-approves-dapagliflozin-to-treatheart-failure-breaking-new-ground-in-sglt2-competition (accessed January 2021).

5. Dhatariya K. Blood ketones: measurement, interpretation, limitations, and utility in the management of diabetic ketoacidosis. Rev Diabet Stud 2016; 13: 217-25.

Publisher's Note Springer Nature remains neutral with regard to jurisdictional claims in published maps and institutional affiliations. 